\title{
LA INFLUENCIA PSICOPEDAGÓGICA PARA LA REAFIRMACIÓN PROFESIONAL EN LA FORMACIÓN DOCENTE POST COVID
}

\author{
A INFLUÊNCIA PSICOPEDAGÓGICA PARA A REAFIRMAÇÃO PROFISSIONAL \\ E A FORMAÇÃO DOCENTE PÓS-COVID
}

\author{
PSYCHOPEDAGOGICAL INFLUENCE FOR PROFESSIONAL REAFFIRMATION \\ IN POST COVID TEACHER TRAINING
}

\author{
Yoisniel Rodríguez Delgado ${ }^{1}$
}

RESUMEN: El Covid - ig trajo como consecuencias el cierre de los diferentes centros educativos y con ellos las iniciativas del reinicio de las clases de forma no presencial mediante el uso de las tecnologías digitales de información, (TIC). Sin embargo con el afán de recuperar los contenidos curriculares, ha sido insuficiente la orientación afectiva - motivacional del estudiante en las nuevas alternativas educativas. E1 objetivo del artículo es sistematizar las influencias psicopedagógicas para la reafirmación profesional en la formación docente post covid. Para la elaboración del mismo se tuvo en cuenta la revisión bibliográfica referente a la problemática a nivel internacional y en el contexto nacional, así como las características de la reafirmación profesional en la formación docente para el tratamiento del objeto en la educación de nivel medio superior en los tiempos de post covid.

Palabras-claves: influencias psicopedagógicas. Reafirmación profesional. Formación docente.

RESUMO: O Covid - I9 resultou no fechamento dos diferentes centros educacionais e com eles as iniciativas para reiniciar as aulas de forma não presencial por meio do uso de tecnologias de informação digital (TIC). No entanto, no esforço de recuperação dos conteúdos curriculares e a orientação afetivo - motivacional do aluno nas novas alternativas educacionais têm sido insuficientes. O objetivo do artigo é sistematizar as influências psicopedagógicas para a reafirmação profissional na formação docente pós COVID. Para a sua elaboração, teve-se em consideração a revisão bibliográfica sobre a problemática a nível internacional e nacional, bem como, as características da reafirmação profissional na formação docente para o tratamento do objeto no ensino secundário superior em tempos pós- pandemia.

Palavras-chave: influências psicopedagógicas. Reafirmação profissional. Treinamento de professor.

\footnotetext{
I Licenciado em Ciéncias da Educą̧ão. Mestrado em Ciências da Educação. Trabalho, professor de Espanhol na Escola pedagógica: Octavio García Hernández. Cienfuegos, Cuba. https://orcid.org/ooooooo2-6745-3368. E-mail: yoisnielr@gmail.com.
} 
ABSTRACT: The Covid - I9 resulted in the closing of the different educational centers and with them the initiatives of the I restart of the classes in a non present way by means of the use of the digital technologies of information, (TIC). however with the desire of recovering the curricular contents, it has been insufficient the affective orientation - the student's motivacional in the new educational alternatives. The objective of the article is to systematize the influences psicopedagógicas for the professional reaffirmation in the educational formation post covid. For the elaboration of the same one one kept in mind the bibliographical revision with respect to the problem at international level and in the national context, as well as the characteristics of the professional reaffirmation in the educational formation for the treatment of the object in the education of half superior level in the times of post covid.

Keywords: you influence psicopedagógicas. Professional Reaffirmation. Educational formation.

\section{INTRODUCCIÓN}

El sistema escolarizado educacional en el mundo se ha visto afectado por la pandemia conocida como el covid - 19, el cual ha puesto en riesgo el cumplimiento de las metas para un desarrollo sostenible como la agenda 2030, el cual plantea... el derecho a la educación para todos y todas, como marco de orientación de políticas nacionales e institucionales.

En cumplimiento a este acuerdo los países trazan acciones educativas desde la utilización de las tecnologías de la información, destacando la educación a distancia en los diferentes niveles educativos.

Si bien estas prácticas han contribuido a minimizar los efectos de la descolarización durante el cierre de las escuelas, ha marcado la desigualdad de condiciones que viven millones, de niños, niñas, adolescentes y jóvenes en el mundo, los cuales no cuentan con las tecnologías adecuadas para recibir las clases a distancia. Otras de las barreras son las carencias de programas educativos en condiciones de catástrofes para evitar las sobrecargas de contenidos curriculares, la fatiga, estrés escolar y las excesivas evaluaciones.

A nivel internacional autores como, Martín L.R. (2020), Argandoña-Mendoza, M. F., Ayón-Parrales, E. B., García-Mejía, R. O., Zambran-Zambrano, Y. A., \& Barcia-Briones, M. F. (2020), corroboran desde la ciencia las consecuencias de la covid 
- I9 en los procesos educativos y marcan las pautas para elaborar acciones desde la diversidad educativa en una etapa post pandémica.

En este contexto, Serey, D., \& Zúñiga, P. (2020), Quevedo-Barros, M., VásquezLafebre, L., Pinzón-Prado, L., \& Quevedo-Vázquez, J. (202I), Hernández-Ramos, J. P., Martínez-Abad, F., \& Sánchez-Prieto, J. C. (202I), trabajan el proceso de formación, sus estudios se dirigen a las competencias profesionales, la convivencia escolar y las influencias de los video tutoriales en los estudiantes.

Sin embargo, desde estas aristas no se concibe los aspectos cognitivos afectivos - motivacional en el proceso de formación, teniendo en cuenta el diagnóstico de los estudiantes.

En Cuba, la investigadora Arteaga, N. L. C. (2020), realiza un análisis sobre la política cubana de enfrentamiento a la covid - ig y el papel de la educación. Si bien el sistema educativo cubano ha puesto en práctica una estrategia de intervención durante la pandemia en la cual se resalta su carácter inclusivo a partir del empleo de una parrilla televisiva, la cual no solo incluye la planificación de clases hacia los diferentes niveles educativos, sino programas formativos complementarios para la formación integral de los estudiantes, esto aparejado a la utilización en menor escala de las tecnologías digitales.

A pesar del interés del gobierno y del Ministerio de Educación en Cuba por aliviar los efectos de la pandemia en la educación de los estudiantes en los diferentes niveles educativos, existen vacíos en las influencias psicopedagógicas para la reafirmación profesional en la formación docente de nivel medio superior.

Por lo que se plantea como objetivo, sistematizar las influencias psicopedagógicas para la reafirmación profesional en la formación docente de nivel medio superior en tiempos de post covid.

\section{FORMACIÓN DOCENTE Y REAFIRMACIÓN PROFESIONAL EN EL NIVEL MEDIO SUPERIOR}

La formación docente debe contribuir a la preparación del sujeto para su accionar en el ejercicio de la profesión desde una concepción humanista, en cual el estudiante sea el protagonista de su propio proceso de formación. 
Autores como: Aquino, (2015), Spech, (2018), Terranova, (2019), Provén, (2019), Villao, (2018), Zapata, (2012), plantean que la formación docente del nivel medio superior se direcciona desde las actividades que se realizan en la institución las cuales se planifican desde un enfoque profesional.

Según Aquino, (2015, p. 3-4) plantea que está constituido por intenciones convertidas en acciones que atraviesen todo el currículo desde ingreso hasta la culminación de estudios. Constituyen abordajes pedagógicos del proceso docente que se realizan con el propósito de aportar conocimientos, habilidades y modos de actuación que tributen eficazmente al perfil del egresado de escuelas pedagógicas.

Por tanto, se asume este criterio, se expresa en la dirección de un sistema de influencias desde lo cognitivo - afectivo motivacional, para formar en el estudiante habilidades e intereses por la profesión y logre su reafirmación profesional desde la práctica educativa para su desempeño en la misma.

Mediante lo planteado, se trabaja la formación docente de nivel medio superior desde un contexto post covid como, proceso sistemático, reflexivo y permanente, mediante las dimensiones cognitivas-afectivas motivacionales, prácticas-valorativas, garantiza la adquisición de conocimientos, habilidades, valores y cualidades profesionales, la cual se expresa en la manera de pensar, percibir, sentir y comportarse del sujeto como personalidad, para dar solución a las contradicciones de la práctica educativa.

De esta manera, Gamboa et, al (2016), concibe la reafirmación profesional como: ...un proceso que se produce en el transcurso del estudio y preparación profesional, esencialmente en los primeros años de los estudiantes de la carrera, a través de la integración y articulación consciente de los agentes educativos de los contextos de actuación significativos dirigidos a la formación y el desarrollo del futuro profesional, de manera que esté apto tanto en lo motivacional como en lo cognitivo para el desempeño e identidad profesional exitosos de su profesión en las condiciones actuales de la escuela. (p. го)

En consecuencia, con el estudio, se coincide que la reafirmación profesional en la formación docente desde un contexto post covid, es un proceso que se realiza a partir de un sistema de influencias pedagógicas, debe mediatizar durante toda la formación 
profesional del sujeto que incluso transciende fuera de la institución formadora, por lo que está presente durante todo el proceso de formación de la personalidad del sujeto. De esta manera, la reafirmación profesional es un proceso formativo porque se identifica con la formación de valores, convicciones, ideales, conocimientos, aptitudes y habilidades que desarrollan la personalidad del estudiante, logra comprometerlos con la tarea que realizan, los hace conscientes de su rol e importancia para la sociedad logrando una satisfacción personal, lo cual contribuye a su autodeterminación profesional.

Para la reafirmación profesional en el proceso de formación de maestros primarios de nivel medio superior el investigador emplea las influencias psicopedagógicas, la cual se manifiesta en el proceso teniendo en cuenta lo afectivo motivacional, cognitivo, actitudinal y valorativo y su integración con las formas organizativas, curriculares y extracurriculares del proceso docente educativo, las cuales constituyen categorías de la dimensión.

Desde estos saberes autores como Rojas (2003), Moreno (2004), García (2010), Urdanivia (2020), emplearon en sus investigaciones lo afectivo-motivacional como categoría psicológica de la motivación.

Desde sus estudios trabajan la motivación hacia la profesión como una tendencia orientadora de la personalidad, cuando el sujeto es capaz de estructurar de forma consciente su motivo profesional, basados en una elaboración y fundamentación consciente de sus propósitos en esta esfera.

De esta manera se tiene en cuenta lo que plantea Caro (2019, p. 4) que '...la motivación inicia produciéndose una etapa de deseo por llegar a una meta, surge interés de la persona por la satisfacción de un motivo '.

Al igual se toma en consideración a los autores, Mendoza Tauler, Leyva Figueredo, Santiesteban Naranjo \& Velázquez Avila (2019, p. 40) cuando plantean que '...la motivación constituye una faceta compleja del proceso docente educativo, por cuanto esta abarca los motivos personales de estudio de los estudiantes”.

Sus aportes radican en crear las condiciones desde el punto de vista teórico, para que los estudiantes comprendan el significado de lo que estudian, sus habilidades, en virtud de lo cual se origina el deseo de conocer lo nuevo. 
Desde esta percepción el autor de la investigación asume la motivación como una categoría para la reafirmación profesional en la formación de maestros primarios de nivel medio superior, por lo que su materialización en el proceso docente educativo se concibe desde la planificación de la actividad, propicia para la estimulación de la motivación de los estudiantes.

El análisis anterior posibilita al investigador definir que la motivación en la reafirmación profesional se dirige a partir de las influencias educativas para ayudar al estudiante a descubrirse así mismo, mediante la exploración de métodos que favorezcan la flexibilidad, la autovaloración y la creatividad. Rodríguez (2019. p.4). Por tanto, para la reafirmación profesional en la formación de maestros primarios de nivel medio superior se debe tener en cuenta la relación cognitiva - práctica valorativa desde la actividad.

Díaz Lazo y Aquino, (2019, p. 6) plantean que la actividad 'es el espacio propicio para la problematización de la relación sujeto-profesión, para establecer relaciones afectivas con los estudiantes que faciliten lograr la relación de ayuda y para demostrar la identificación con la profesionalidad del docente que la imparte."

La idea anterior permite, ratificar la importancia de la actividad como espacio que debe ser aprovechado desde el punto de vista pedagógico para fomentar; emociones, sentimientos, vivencias y regular conductas con respecto a la profesión elegida, a partir de la preparación del profesor en el contenido, de las habilidades para el trabajo en grupo y su ejemplo personal.

Por consiguiente, las influencias psicopedagógicas se expresan en las actividades que se establecen en las relaciones de acondicionamiento entre los procesos cognoscitivos, prácticos y valorativos de la enseñanza, realizándose una articulación dialéctica entre los factores que intervienen e influyen y permiten determinar el significado profesional de la dirección del proceso docente educativo.

Constituye según González (1994) un nivel donde se integra la motivación profesional como formación psíquica relacionada con el conocimiento del contenido de la profesión.

Urdanivia (2020, p.31) ... alega que la práctica como actividad, permite al estudiante actuar con el objeto de la formación, mediada por un sistema de relaciones 
que se estable con sus estudiantes. De esta manera es criterio del autor que la práctica como actividad pedagógica se desarrolla en el contexto, expresa un significado e intencionalidad formativa en el estudiante, sin embargo, se considera que las influencias que se establecen entre las relaciones de los factores que intervienen en las mismas han sido poco trabajadas en la formación de maestros primarios de nivel medio superior, donde lo valorativo facilita el intercambio de ideas, opiniones a partir de métodos reflexivos los estudiantes se puedan apropiar de todo un sistema de influencias lo cual se traduce en su forma de pensar, ser y actuar.

Desde este análisis el investigador aborda las categorías para ejercer un sistema de influencias psicopedagógicas como dimensión para la reafirmación profesional en la formación de maestros primarios de nivel medio superior en un contexto post covid. I. Afectivo-motivacional, se trabajan en los motivos profesionales que los estudiantes presentan por la profesión, puesto que los estudiantes vienen con preconceptos ya formados por experiencias anteriores en las orientaciones recibidas, para mediante la participación en las actividades poder estimular su motivación por la especialidad.

2. Cognitivo, se concibe a partir de la intencionalidad educativa en el proceso para la reafirmación profesional en el que se manifiesta el Ser, Saber, Saber Hacer, para el desarrollo del conocimiento y habilidades profesionales, donde el estudiante se apropia de la cultura y los procederes que se les transmite.

Contenidos conceptuales del Ser: Estos contenidos hacen referencia a los valores que forman parte de los componentes cognitivos (conocimientos, creencias, experiencias) de los componentes afectivos (sentimientos, aspiraciones, deseos) y los componentes de comportamientos que se observan en sus interrelaciones con sus pares $\mathrm{u}$ otros factores que intervienen en el proceso.

Saber: Se refiere al aprendizaje del estudiante en cuanto a la cultura que se le ha transmitido, la apropiación de conocimientos y habilidades profesionales.

Saber Hacer: Se considera dentro de los contenidos procedimentales de las acciones, modos de actuar, afrontar, plantear y resolver situaciones problémicas que se puedan presentar en su formación o actividad profesional.

3. Actitudinal y valorativo, se muestra en el compromiso del estudiante en su desempeño por la actividad pedagógica, en la búsqueda de información y participación 
sistemática del estudiante, en la relación con los otros estudiantes, profesores, y factores que intervienen en su formación, puesto que se deben crear desde el contexto situaciones prácticas para analizar sus manifestaciones y procederes.

Estas categorías se integran en el proceso de formación docente a partir de las siguientes cuestiones desde una perspectiva pedagógica:

Organizativas: se deben crear ambientes y espacios agradables, fuera de lo tradicional, donde los estudiantes se sientan con la libertad de intercambiar opiniones, ideas, donde fluya una intercomunicación abierta sin ruidos, interrupciones, evitar el enjuiciamiento, la crítica.

Curriculares: el centro debe elaborar un proyecto curricular sistemático, planificado, flexible, dinámico, participativo que comprende las actividades pedagógicas de forma sistémica para lograr la satisfacción profesional, personal y el cumplimiento del perfil del egresado.

De esta manera el contenido debe intencional la reafirmación profesional de los estudiantes desde la estimulación, el incentivo, la creatividad y la motivación. Debe promover el espacio para que los estudiantes apliquen sus saberes en la práctica, donde se produce un intercambio de retroalimentación, aplica el contenido aprendido y se nutre de nuevos conocimientos que conducen al desarrollo, cumpliendo con una categoría pedagógica fundamental en el proceso de investigación, formación y desarrollo.

Extracurriculares: Se refiere a las actividades para la reafirmación profesional de manera intencional las relaciones profesor - estudiante, tutor - estudiante, estudiante - estudiante y estudiante - comunidad.

Desde estas consideraciones se concibe la integración de los aspectos psicológicos y pedagógicos, encierra un nivel superior de sistematización, revelándose en la unidad, funcionamiento y desarrollo de los factores internos y externos de la personalidad del sujeto como fundamento de la reafirmación profesional.

Esta idea pone en primer plano de integración a la relación entre la educación como factor y función de la práctica social y el desarrollo de la personalidad de los estudiantes, relación entre la formación y desarrollo como expresión particular de la reafirmación profesional como proceso pedagógico. 


\section{CONSIDERACIONES FINALES}

Las influencias psicopedagógicas dirigidas mediante un currículo planificado, sistemático, flexible y dinámico favorece la reafirmación profesional en la formación docente en un contexto post covid.

\section{REFERENCIAS BIBLIOGRÁFICAS}

Aquino, G. (2015). La orientación vocacional profesional pedagógica desde la clase de Introducción a la Didáctica general. Clase Metodológica Instructiva para las Escuelas Pedagógicas. UNISS. Material Impreso. pág. 3 y 4., Sancti Spíritus, Cuba.

Argandoña-Mendoza, M. F., Ayón-Parrales, E. B., García-Mejía, R. O., Zambran Zambrano, Y. A., \& Barcia-Briones, M. F. (2020). La educación en tiempo de pandemia. Un reto Psicopedagógico para el docente. Polo del Conocimiento, 5(7), 819 848 .

Aristigui. N. (2015). La reafirmación profesional pedagógica en la universidad, su Impacto social. Multimed.

Arteaga, N. L. C. (2020). La política cubana frente a la covidig. La educación en este contexto. Revista Boletín Redipe, 9(8), 24-34.

Bell-Speck, Y. (2018). Retos y perspectivas en la preparación del docente de la Escuela Pedagógica.

Caro, Y., Cueto, R., \& Sánchez, S. (2019). Hacia el enfoque familiar de la orientación profesional pedagógica. Mendive. Revista de Educación, I7(I), 69-83.

Díaz R., C., Lazo, M. \& Aquino, G. (2019): “Tareas docentes desde las clases de fundamentos de pedagogía para orientar hacia la carrera de Maestros Primarios ”, Revista Atlante: Cuadernos de Educación y Desarrollo (enero 2019). En línea García, Y. (2010). Metodología para el diagnóstico de la Reafirmación Profesional Pedagógica

en la formación del personal docente (Doctoral dissertation, Tesis de maestría inédita. Camagüey: Universidad de Ciencias Pedagógicas "José Martí).

Hernández-Ramos, J. P., Martínez-Abad, F., \& Sánchez-Prieto, J. C. (202I). El empleo de videotutoriales en la era post COVIDı: valoración e influencia en la identidad docente del futuro profesional. Revista de Educación a Distancia (RED), 2I(65). 
Martín, R. L. (2020). Reflexiones educativas para el posCOVID-19. Recordando el futuro. Revista Internacional de Educación para la Justicia Social, 9(3), I27-I40.

Mendoza, L., Leyva, P., Santiesteban, E., \& Velázquez, K. (2019). Estimulación de la motivación: una alternativa para lograr su desarrollo.

Quevedo-Barros, M., Vásquez-Lafebre, L., Pinzón-Prado, L., \& Quevedo-Vázquez, J. (202I). Gerencia del talento humano basado en competencias post covid-I9. Revista Científica FIPCAEC (Fomento de la investigación y publicación en Ciencias Administrativas, Económicas y Contables). ISSN: 2588-09oX. Polo de Capacitación, Investigación y Publicación (POCAIP), 6(3), 674-683.

Rodríguez, Y. (2019). La reafirmación profesión pedagógica en la formación docente de nivel medio superior. Revista Conrado, 15 (70).

Rojas, A. (2003). La formación vocacional hacia la carrera Licenciatura en Educación Preescolar: Una propuesta pedagógica (doctoral dissertativa, Tesis presentada para la obtención del grado científico de Doctor en Ciencias Pedagógicas. Cienfuegos, Cuba) Serey, D., \& Zúñiga, P. (2020). La convivencia escolar post COVID i9: una propuesta didáctica desde el coaching educativo.

Terranova, R. (2019). La práctica preprofesional integradora. Editorial Académica Universitaria (Edacun). Universidad de Las Tunas. Cuba.

Villao, S. (2018). Modelo de Profesionalización Pedagógica de los docentes universitarios para la Gestión del Proceso de Enseñanza-Aprendizaje. Revista Electrónica Formación y Calidad Educativa. Vol. 6, (3) Zapata, G. Zúñiga, G. \& Rodríguez, R. (2012). La formación de los docentes del nivel medio superior Escuela Pública. Revista Iberoamericana para la Investigación y el Desarrollo Educativo. (9) 